\section{0-014 PRETERM PIGLETS DISPLAY IMPAIRED PHYSICAL ACTIVITY AND ALTERED BEHAVIOUR DURING THE FIRST WEEKS OF LIFE}

'AD Andersen, ${ }^{1} \mathrm{SL}$ Munch, ${ }^{1} \mathrm{PT}$ Sangild, ${ }^{2} \mathrm{JD}$ Lund, ${ }^{1} \mathrm{~T}$ Thymann. ${ }^{1}$ Clinical and Experimental Nutrition, University of Copenhagen, Frederiksberg C, Denmark; ${ }^{2} A R L A$, Food Ingredients, Viby J, Denmark

\subsection{6/archdischild-2014-307384.82}

Background and aims Premature birth interrupts normal growth and may affect postnatal brain development. We hypothesised that prematurity in pigs would affect neuromuscular control and behaviour also beyond the neonatal period.

Methods Caesarean-delivered preterm ( $\mathrm{n}=44,90 \%$ gestation) and term ( $\mathrm{n}=33,100 \%$ gestation) piglets were fed parenterally for five days and then enterally with milk-replacer until $\mathrm{d} 26$. Time until basic motor skill (BMS) acquisition (eye lid opening, first walk and stand) were recorded, coordination assessed, and locomotion and general exploration were tracked from open field video recordings on $\mathrm{d} 4, \mathrm{~d} 9, \mathrm{~d} 16$ and $\mathrm{d} 23$. A novel-object recognition test was performed on $\mathrm{d} 24$ (assessing both specific exploration and short-term memory), and learning ability was assessed with a clicker-based poke-reward test from $\mathrm{d} 18-\mathrm{d} 25$ in a subset of piglets.

Results BMS acquisition was delayed in preterm piglets (all $\mathrm{p}<$ 0.001). Coordination scores were lower in preterm piglets at all ages whereas locomotion and exploration were reduced only on $\mathrm{d} 4$ (all $\mathrm{p}<0.05)$. Preterm piglets explored novel objects less ( $\mathrm{p}$ $<0.001$ ) but short-term memory assessments were not different. Poke-reward performance improved over time in both preterm and term pigs but did not differ significantly between groups, which partly reflects that only clinically healthy preterm piglets could be tested. In preterm piglets, locomotion on $\mathrm{d} 23$ was increased $(\mathrm{p}<0.01)$ when parenteral nutrition had been supplemented with enteral nutrition the first five days after birth.

Conclusion Acquisition of neuromuscular control, locomotion and exploration are quantifiable functional neurological endpoints in preterm piglets that may be used to characterise developmental disturbances and nutritional interventions.

\section{0-015 TEMPORARY THERAPEUTIC WINDOW OF CANNABIDIOL EXTENDS BEYOND 12 HOURS IN HYPOXIC-ISCHAEMIC NEWBORN MICE}

${ }^{1} \mathrm{~N}$ ElDemerdash, ${ }^{2} \mathrm{MR}$ Pazos, ${ }^{2} \mathrm{M}$ Ceprián, ${ }^{2} \mathrm{~L}$ Jiménez, ${ }^{3}$ OlaD Saugstad, ${ }^{2}$ JOSE MartinezOrgado. ${ }^{1}$ Nueropediatria, FBl-Hospital Universitrio Puerta de Hierro, Department of Pediatric Research, Oslo University Hospital, University of Oslo Norway, Madrid, Spain; ${ }^{2}$ Nueropediatria, FBI-Hospital Universitrio Puerta de Hierro, Madrid, Spain; ${ }^{3}$ Pediatrics, Department of Pediatric Research, Oslo University Hospital, University of Oslo Norway, Oslo, Norway

\subsection{6/archdischild-2014-307384.83}

Background and aim cannabidiol (CBD) leads to significant and long-term sustained neuroprotection in hypoxic-ischaemic (HI) newborn rodents. We aim to determine the temporary therapeutic window (TTW) of CBD. Such TTW is stablished in $6 \mathrm{~h}$ for the standard therapy, hypothermia.

Methods 9-day old C57BL6 mice underwent a HI insult (10\% oxygen for $90 \mathrm{~min}$ after left carotid artery electrocoagulation). Then, $0.1 \mathrm{~mL}$ of vehicle (ethanol:solutol:saline 2:1:17) (HV, $\mathrm{n}=25)$ or CBD $(1 \mathrm{mg} / \mathrm{kg})$ was administered s.c. $15 \mathrm{~min}$, or 1 , $3,6,12$ or $24 \mathrm{~h}$ after the end of the HI insult (HC0.15 $\mathrm{n}=10$; $\mathrm{HC} 1, \mathrm{n}=10 ; \mathrm{HC} 3, \mathrm{n}=10 ; \mathrm{HC} 6, \mathrm{n}=10 ; \mathrm{HC} 12, \mathrm{n}=10$; HC24, $\mathrm{n}=9$, respectively). Seven days later MRI scan (T2W) were carried out in formaline-included pup brains (ipsilateral hemisphere volume loss, IVHL), whereas the penumbral perilesional area (parieto-occipital cortex) was studied using Nissl staining (necrotic damage, by a neuropathological score, NPS), TUNEL staining (apoptotic damage) and GFAP immunohistochemistry (astrocyte viability). Non-HI mice served as controls (SHM, $\mathrm{n}=15)$.

Results CBD, administered up to $12 \mathrm{~h}$ after HI, showed a significant neuroprotective effect, reducing HI-induced IHVL and NPS by $60 \%$, TUNEL + count by $90 \%$ and astrocyte damage by $50 \%$. When CBD administration was delayed $24 \mathrm{~h}$, however, mild neuroprotective effect was still observable regarding NPS or TUNEL, but not IVH loss or astrocyte viability

Conclusions TTW for CBD seems to be between 12 and $24 \mathrm{~h}$ after the end of the HI insult.

Supported by FIS PS09/01900, Health Trust South East Norway and GWCRI091190-2.

\section{0-015a IMPROVED DEVELOPMENT OF CULTURED OLIGODENDROGLIA AT 5\% IN COMPARISON TO $21 \%$ OXYGEN}

C Brill, G Weikert, R Herrmann, C Bührer, S Endesfelder, T Schmitz. Neonatology, Charité University Hospital Berlin, Berlin, Germany

\subsection{6/archdischild-2014-307384.84}

Immature oligodendroglia of the developing brain are highly susceptible to hyperoxia. As the in-vivo $\mathrm{O}_{2}$ saturation of the brain parenchyma is about $3-7 \%$, we hypothesised that the $21 \% \mathrm{O}_{2}$ commonly sustained in cell culture poses a hyperoxic challenge to immature oligodendroglia affecting their development.

We cultured primary rat oligodendroglial precursor cells (OPC) for $24 \mathrm{~h}, 48 \mathrm{~h}$ and $96 \mathrm{~h}$ at $5 \%$ and $21 \%$ and assessed their developmental progress through analysis of cell numbers of different oligodendroglial stages. For immunocytochemistry, $\mathrm{A} 2 \mathrm{~B} 5+, \mathrm{O} 4+$, and $\mathrm{O} 1+$ markers were used to label precursor, immature and mature stages respectively, combined with Ki67 for proliferation or TUNEL for apoptosis. qPCR was used to determine gene expression of factors important for development (Olig1, Olig2, Sox9, Sox10) and maturation (MBP, CNP). NRF2 and SOD2 expression was measured to quantify responses to oxidative stress.

Morphology of OLC varied greatly between 21\% and 5\% at all time points. At 48 h, O4 positive immature oligodendroglia frequently had multiple processes with typical shape at $5 \%$ but rarely at $21 \%$ oxygen. Gene expression of MBP, CNP, Olig1, and Olig2 was significantly reduced and antioxidant genes NRF2 and SOD2 were significantly up-regulated after $48 \mathrm{~h}$ at $21 \%$ immunohistochemistry (each $\mathrm{p}<0.05$ ). However, there was no difference in cell death as analysed by NG2+TUNEL+ OPCs.

Altogether, these findings indicate that $21 \% \mathrm{O}_{2}$ in vitro has negative effects on oligodendroglial development. It has to be debated whether $5 \% \mathrm{O}_{2}$ resemble physiological oxygen conditions more appropriately than current standard protocols with $21 \%$ for oligodendroglial cultures. 